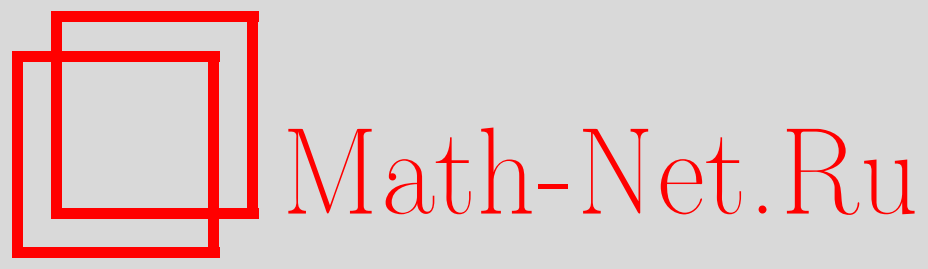

Т. А. Суслина, Усреднение параболической задачи Коши в классе Соболева $H^{1}\left(\mathbb{R}^{d}\right)$, Функи. анализ и его прил., 2010, том 44, выпуск 4, 91-96

DOI: https://doi.org/10.4213/faa3017

Использование Общероссийского математического портала Math$\mathrm{Net.Ru}$ подразумевает, что вы прочитали и согласны с пользовательским соглашением http://www.mathnet.ru/rus/agreement

Параметры загрузки:

IP : 3.85 .183 .62

26 апреля 2023 г., $17: 22: 35$

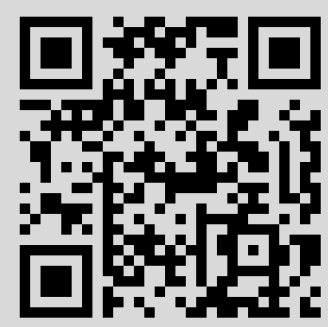


УДК 517.956.4

\section{Усреднение параболической задачи Коши в классе Соболева $H^{1}\left(\mathbb{R}^{d}\right)$}

(c) 2010. Т. А. СуслинА*

Светлой памяти дорогого Учителя Михаила Шлемовича Бирмана

1. Введение. Рассматривается задача об усреднении в пределе малого периода (гомогенизации) решения $\mathbf{u}_{\varepsilon}$ задачи Коши для параболического уравнения в $\mathbb{R}^{d}$ с быстро осциллирующими коэффициентами (см. ниже $\left.(2),(8)\right)$. Параметр $\varepsilon$ имеет смысл малого периода. Задачи гомогенизации для параболических уравнений активно изучались традиционными методами теории усреднений (см., например, книги [1]-[3]). Соответствующие результаты дают сходимость (в подходящем функциональном классе) решения $\mathbf{u}_{\varepsilon}$ к решению $\mathbf{u}_{0}$ усредненной (эффективной) задачи с постоянными коэффициентами. К эллиптическим и параболическим задачам теории усреднений применялся также спектральный подход (см. [4], [5]).

Мы используем новый теоретико-операторный подход, развитый применительно к эллиптическим задачам в [6]-[9]. С помощью этого подхода в [10], [11] была получена не только сходимость решения $\mathbf{u}_{\varepsilon} \quad \mathrm{K} \mathbf{u}_{0}$ в $L_{2}\left(\mathbb{R}^{d}\right.$ ) (при фиксированном времени $\tau$ ), но и оценка порядка $\varepsilon$ для $L_{2}$-нормы разности $\mathbf{u}_{\varepsilon}-\mathbf{u}_{0}$, равномерная относительно $L_{2}$-нормы начальных данных (см. ниже оценку $\left.(5)\right)$. В дальнейшем в [12] тем же методом была получена более точная аппроксимация решения в $L_{2}\left(\mathbb{R}^{d}\right)$ с погрешностью $O\left(\varepsilon^{2}\right) ;$ при этом учитывался корректор. Цель настоящей работь - получить аппроксимацию решения в пространстве Соболева $H^{1}\left(\mathbb{R}^{d}\right)$, равномерную относительно $L_{2}$-нормы начальных данных, с погрешностью $O(\varepsilon)$.

Другой подход к получению результатов подобного типа был предложен в [13], [14] применительно к эллиптическим задачам и в [15] применительно к параболическим задачам.

2. Постановка задачи. Пусть $\Gamma-$ некоторая решетка в $\mathbb{R}^{d}$. Через $\Omega$ обозначим элементарную ячейку решетки $\Gamma$. Ниже через $\widetilde{\Gamma}$ обозначается решетка, двойственная к $\Gamma$, а через $\widetilde{\Omega}-$ зона Бриллюэна решетки $\widetilde{\Gamma}$. Для всякой Г-периодической функции $\varphi(\mathbf{x})$ используем обозначение $\varphi^{\varepsilon}(\mathbf{x})=\varphi(\mathbf{x} / \varepsilon), \varepsilon>0$.

Через $L_{2}\left(\mathbb{R}^{d} ; \mathbb{C}^{n}\right)$ обозначим пространство $\mathbb{C}^{n}$-значных квадратично суммируемых функций в $\mathbb{R}^{d}$. Пусть $m, n \in \mathbb{N}, m \geqslant n$, и $b(\mathbf{D})$ есть $(m \times n)$-матричный однородный дифференциальный оператор первого порядка с постоянными коэффициентами. Здесь $\mathbf{D}=-i \nabla$. Оператору $b(\mathbf{D})$ отвечает символ $-(m \times n)$ матрица $b(\boldsymbol{\xi})$, являющаяся линейной однородной функцией от $\boldsymbol{\xi} \in \mathbb{R}^{d}$. Предполагается, что $\operatorname{rank} b(\boldsymbol{\xi})=n$ при $\boldsymbol{\xi} \neq 0$. Это равносильно выполнению неравенств

$$
\alpha_{0} \mathbf{1}_{n} \leqslant b(\boldsymbol{\theta})^{*} b(\boldsymbol{\theta}) \leqslant \alpha_{1} \mathbf{1}_{n}, \quad|\boldsymbol{\theta}|=1,0<\alpha_{0} \leqslant \alpha_{1}<\infty,
$$

* Работа выполнена при поддержке РФФИ (грант 08-01-00209-а) и Программы поддержки ведущих научных школ (грант НШ-5931.2010.1). 
с некоторыми постоянными $\alpha_{0}, \alpha_{1}$. Далее, пусть $(m \times m)$-матрица-функция $g(\mathbf{x})$ и $(n \times n)$-матрица-функция $Q(\mathbf{x})$ являются ограниченными, положительно определенными и периодическими относительно решетки $\Gamma$.

Рассмотрим следующую задачу Коши для функции $\mathbf{u}_{\varepsilon}(\mathbf{x}, \tau), \mathbf{x} \in \mathbb{R}^{d}, \tau \geqslant 0$ :

$$
Q^{\varepsilon}(\mathbf{x}) \frac{\partial \mathbf{u}_{\varepsilon}(\mathbf{x}, \tau)}{\partial \tau}=-b(\mathbf{D})^{*} g^{\varepsilon}(\mathbf{x}) b(\mathbf{D}) \mathbf{u}_{\varepsilon}(\mathbf{x}, \tau), \quad Q^{\varepsilon}(\mathbf{x}) \mathbf{u}_{\varepsilon}(\mathbf{x}, 0)=\phi(\mathbf{x}),
$$

где $\phi \in L_{2}\left(\mathbb{R}^{d} ; \mathbb{C}^{n}\right)$. Нас интересует поведение решения при малом $\varepsilon$.

3. Старший член аппроксимации решения $\mathbf{u}_{\varepsilon}$. При $\varepsilon \rightarrow 0$ решение задачи (2) сходится (в некотором смысле) к решению эффективной задачи

$$
\bar{Q} \frac{\partial \mathbf{u}_{0}(\mathbf{x}, \tau)}{\partial \tau}=-b(\mathbf{D})^{*} g^{0} b(\mathbf{D}) \mathbf{u}_{0}(\mathbf{x}, \tau), \quad \bar{Q} \mathbf{u}_{0}(\mathbf{x}, 0)=\phi(\mathbf{x}) .
$$

Здесь $\bar{Q}=|\Omega|^{-1} \int_{\Omega} Q(\mathbf{x}) d \mathbf{x}$, а $g^{0}-$ положительная $(m \times m)$-матрица, называемая эффективной матрицей. Для описания $g^{0}$ введем Г-периодическую $(n \times m)$ матрицу-функцию $\Lambda(\mathbf{x})$, являющуюся (слабым) решением задачи

$$
b(\mathbf{D})^{*} g(\mathbf{x})\left(b(\mathbf{D}) \Lambda(\mathbf{x})+\mathbf{1}_{m}\right)=0, \quad \int_{\Omega} \Lambda(\mathbf{x}) d \mathbf{x}=0 .
$$

Здесь $\mathbf{1}_{m}-$ единичная $(m \times m)$-матрица. Далее, введем $(m \times m)$-матрицуфункцию $\tilde{g}(\mathbf{x})=g(\mathbf{x})\left(b(\mathbf{D}) \Lambda(\mathbf{x})+\mathbf{1}_{m}\right)$. Тогда $g^{0}=|\Omega|^{-1} \int_{\Omega} \tilde{g}(\mathbf{x}) d \mathbf{x}$. Отметим сразу оценки $\underline{g} \leqslant g^{0} \leqslant \bar{g}$, известные как вилка Фойгта-Рейсса. Здесь

$$
\bar{g}=|\Omega|^{-1} \int_{\Omega} g(\mathbf{x}) d \mathbf{x}, \quad(\underline{g})^{-1}=|\Omega|^{-1} \int_{\Omega} g(\mathbf{x})^{-1} d \mathbf{x} .
$$

В [10], [11] был установлен следующий результат.

Теорема 1. При фиксированном $\tau>0$ решение $\mathbf{u}_{\varepsilon}(\cdot, \tau)$ задачи $(2)$ сходится $n р и \varepsilon \rightarrow 0$ в $L_{2}\left(\mathbb{R}^{d} ; \mathbb{C}^{n}\right) \kappa$ решению $\mathbf{u}_{0}(\cdot, \tau)$ эффективной задачи $(3)$. При этом

$$
\left\|\mathbf{u}_{\varepsilon}(\cdot, \tau)-\mathbf{u}_{0}(\cdot, \tau)\right\|_{L_{2}\left(\mathbb{R}^{d}\right)} \leqslant C_{1} \varepsilon \tau^{-1 / 2}\|\phi\|_{L_{2}\left(\mathbb{R}^{d}\right)}, \quad \varepsilon>0, \tau>0 .
$$

Постоянная $C_{1}$ зависит лишь от норм $\|g\|_{L_{\infty}},\left\|g^{-1}\right\|_{L_{\infty}},\|Q\|_{L_{\infty}},\left\|Q^{-1}\right\|_{L_{\infty}}$, от постоянных $\alpha_{0}, \alpha_{1}$ из (1) и от параметров решетки $\Gamma$.

4. Аппроксимация решения в классе $\boldsymbol{H}^{\mathbf{1}}$. Сейчас мы укажем аппроксимацию решения $\mathbf{u}_{\varepsilon}(\cdot, \tau)$ при фиксированном $\tau>0$ по норме в пространстве Соболева $H^{1}\left(\mathbb{R}^{d} ; \mathbb{C}^{n}\right)$. В аппроксимации учитывается член первого порядка по $\varepsilon$ - так называемый корректор. Кроме того, приводится аппроксимация в $L_{2}\left(\mathbb{R}^{d} ; \mathbb{C}^{m}\right)$ для потока $\mathbf{p}_{\varepsilon}=g^{\varepsilon} b(\mathbf{D}) \mathbf{u}_{\varepsilon}$.

Теорема 2. Пусть $\mathbf{u}_{\varepsilon}-$ решение задачи (2) и $\mathbf{u}_{0}-$ решение задачи (3). Тогда при $\tau>0$ и $0<\varepsilon \leqslant \tau^{1 / 2}$ справедливы оченки

$$
\begin{aligned}
&\left\|\mathbf{u}_{\varepsilon}(\cdot, \tau)-\mathbf{u}_{0}(\cdot, \tau)-\varepsilon \Lambda^{\varepsilon} b(\mathbf{D}) \mathbf{u}_{0}(\cdot, \tau)\right\|_{H^{1}\left(\mathbb{R}^{d}\right)} \leqslant C(\tau) \varepsilon\|\phi\|_{L_{2}\left(\mathbb{R}^{d}\right)} \\
&\left\|g^{\varepsilon} b(\mathbf{D}) \mathbf{u}_{\varepsilon}(\cdot, \tau)-\tilde{g}^{\varepsilon} b(\mathbf{D}) \mathbf{u}_{0}(\cdot, \tau)\right\|_{L_{2}\left(\mathbb{R}^{d}\right)} \leqslant C_{4} \tau^{-1} \varepsilon\|\boldsymbol{\phi}\|_{L_{2}\left(\mathbb{R}^{d}\right)}
\end{aligned}
$$

Здесъ $C(\tau)=C_{2} \tau^{-1}+C_{3} \tau^{-1 / 2}$. Постояннье $C_{2}, C_{3}, C_{4}$ зависят лишь от $d, m$, $n,\|g\|_{L_{\infty}},\left\|g^{-1}\right\|_{L_{\infty}},\|Q\|_{L_{\infty}},\left\|Q^{-1}\right\|_{L_{\infty}}, \alpha_{0}, \alpha_{1}$ и от параметров решетки $\Gamma$. 
5. Задача Коши для неоднородного уравнения. Пусть $0<T \leqslant \infty$. Рассмотрим следующую задачу Коши для функции $\mathbf{v}_{\varepsilon}(\mathbf{x}, \tau), \mathbf{x} \in \mathbb{R}^{d}, \tau \in(0, T)$ : $Q^{\varepsilon}(\mathbf{x}) \frac{\partial \mathbf{v}_{\varepsilon}(\mathbf{x}, \tau)}{\partial \tau}=-b(\mathbf{D})^{*} g^{\varepsilon}(\mathbf{x}) b(\mathbf{D}) \mathbf{v}_{\varepsilon}(\mathbf{x}, \tau)+\mathbf{F}(\mathbf{x}, \tau), \quad Q^{\varepsilon}(\mathbf{x}) \mathbf{v}_{\varepsilon}(\mathbf{x}, 0)=\phi(\mathbf{x})$,

где $\phi \in L_{2}\left(\mathbb{R}^{d} ; \mathbb{C}^{n}\right), \mathbf{F} \in \mathscr{H}_{p}(T), 1<p \leqslant \infty$. Здесь использовано обозначение $\mathscr{H}_{p}(T)=L_{p}\left((0, T) ; L_{2}\left(\mathbb{R}^{d} ; \mathbb{C}^{n}\right)\right)$. Положим

$$
\begin{aligned}
&\|\mathbf{F}\|_{\mathscr{H}_{p}(T)}^{p}=\int_{0}^{T}\|\mathbf{F}(\cdot, \tau)\|_{L_{2}\left(\mathbb{R}^{d}\right)}^{p} d \tau, \quad 1<p<\infty, \\
&\|\mathbf{F}\|_{\mathscr{H}_{\infty}(T)}=\underset{0<\tau<T}{\operatorname{ess-\operatorname {sup}}}\|\mathbf{F}(\cdot, \tau)\|_{L_{2}\left(\mathbb{R}^{d}\right)} .
\end{aligned}
$$

Пусть $\mathbf{v}_{0}(\mathbf{x}, \tau), \mathbf{x} \in \mathbb{R}^{d}, \tau \in(0, T)$, - решение эффективной задачи

$$
\bar{Q} \frac{\partial \mathbf{v}_{0}(\mathbf{x}, \tau)}{\partial \tau}=-b(\mathbf{D})^{*} g^{0} b(\mathbf{D}) \mathbf{v}_{0}(\mathbf{x}, \tau)+\mathbf{F}(\mathbf{x}, \tau), \quad \bar{Q} \mathbf{v}_{0}(\mathbf{x}, 0)=\phi(\mathbf{x})
$$

В [11] был получен следующий результат.

Теорема 3. Пусть $\phi \in L_{2}\left(\mathbb{R}^{d} ; \mathbb{C}^{n}\right)$ u $\mathbf{F} \in \mathscr{H}_{p}(T), 1<p \leqslant \infty$. При фиксированном $\tau \in(0, T)$ решение $\mathbf{v}_{\varepsilon}(\cdot, \tau)$ задачи $(8)$ сходится $п р и \varepsilon \rightarrow 0$ в $L_{2}\left(\mathbb{R}^{d} ; \mathbb{C}^{n}\right)$ $\kappa$ решению $\mathbf{v}_{0}(\cdot, \tau)$ эффективной задачи (9). При $\tau \in(0, T)$ и $\varepsilon>0$ справедлива оченка

$$
\begin{gathered}
\left\|\mathbf{v}_{\varepsilon}(\cdot, \tau)-\mathbf{v}_{0}(\cdot, \tau)\right\|_{L_{2}\left(\mathbb{R}^{d}\right)} \leqslant C_{1}\left(\varepsilon \tau^{-1 / 2}\|\phi\|_{L_{2}\left(\mathbb{R}^{d}\right)}+\theta_{p}(\varepsilon, \tau)\|\mathbf{F}(\cdot, \tau)\|_{\mathscr{H}_{p}(\tau)}\right), \\
\theta_{p}(\varepsilon, \tau)= \begin{cases}c_{p} \varepsilon(\tau+1)^{1 / 2-1 / p}, & 2<p \leqslant \infty, \\
\varepsilon(\ln (\tau+1)+2|\ln \varepsilon|)^{1 / 2}, & p=2, \\
\check{c}_{p} \varepsilon^{2-2 / p}, & 1<p<2 .\end{cases}
\end{gathered}
$$

Отметим, что при фиксированном $\tau$ коэффициент при $\|\mathbf{F}\|_{\mathscr{H}_{p}}$ в (10) есть $O(\varepsilon)$ при $p>2, O\left(\varepsilon|\ln \varepsilon|^{1 / 2}\right)$ при $p=2$ и $O\left(\varepsilon^{2-2 / p}\right)$ при $1<p<2$.

Теперь мы укажем аппроксимацию решения $\mathbf{v}_{\varepsilon}$ по норме в $H^{1}\left(\mathbb{R}^{d} ; \mathbb{C}^{n}\right)$ и аппроксимацию соответствующего потока в $L_{2}\left(\mathbb{R}^{d} ; \mathbb{C}^{m}\right)$. Для формулировки результата нам понадобится вспомогательный сглаживающий оператор $\Pi_{\varepsilon}$, действующий в $L_{2}\left(\mathbb{R}^{d} ; \mathbb{C}^{n}\right)$ и определенный соотношением

$$
\left(\Pi_{\varepsilon} \mathbf{u}\right)(\mathbf{x})=(2 \pi)^{-d / 2} \int_{\widetilde{\Omega} / \varepsilon} \widehat{\mathbf{u}}(\boldsymbol{\xi}) e^{i \mathbf{x} \boldsymbol{\xi}} d \boldsymbol{\xi} .
$$

Здесь $\widehat{\mathbf{u}}(\boldsymbol{\xi})$ - Фурье-образ функции $\mathbf{u}(\mathbf{x})$.

Теорема 4. Пусть $\mathbf{v}_{\varepsilon}$ - решение задачи (8) при $\boldsymbol{\phi} \in L_{2}\left(\mathbb{R}^{d} ; \mathbb{C}^{n}\right)$ u $\mathbf{F} \in \mathscr{H}_{p}(T)$, $2<p \leqslant \infty$. Пусть $\mathbf{v}_{0}$ - решение задачи (9). Тогда при $\tau \in(0, T) u 0<\varepsilon \leqslant \tau^{1 / 2}$ справедливы оценки

$$
\begin{aligned}
& \left\|\mathbf{v}_{\varepsilon}(\cdot, \tau)-\mathbf{v}_{0}(\cdot, \tau)-\varepsilon \Lambda^{\varepsilon} b(\mathbf{D}) \Pi_{\varepsilon} \mathbf{v}_{0}(\cdot, \tau)\right\|_{H^{1}\left(\mathbb{R}^{d}\right)} \\
& \quad \leqslant C^{\prime}(\tau) \varepsilon\|\phi\|_{L_{2}\left(\mathbb{R}^{d}\right)}+\rho_{p}(\varepsilon, \tau)\|\mathbf{F}(\cdot, \tau)\|_{\mathscr{H}_{p}(\tau)}, \\
& \left\|g^{\varepsilon} b(\mathbf{D}) \mathbf{v}_{\varepsilon}(\cdot, \tau)-\tilde{g}^{\varepsilon} b(\mathbf{D}) \Pi_{\varepsilon} \mathbf{v}_{0}(\cdot, \tau)\right\|_{L_{2}\left(\mathbb{R}^{d}\right)} \\
& \quad \leqslant C_{4}^{\prime} \tau^{-1} \varepsilon\|\phi\|_{L_{2}\left(\mathbb{R}^{d}\right)}+\widetilde{\rho}_{p}(\varepsilon, \tau)\|\mathbf{F}(\cdot, \tau)\|_{\mathscr{H}_{p}(\tau)} .
\end{aligned}
$$


Здесъ $C^{\prime}(\tau)=C_{2}^{\prime} \tau^{-1}+C_{3}^{\prime} \tau^{-1 / 2}$,

$$
\begin{aligned}
& \rho_{p}(\varepsilon, \tau)= \begin{cases}C_{5} \varepsilon^{1-2 / p}+C_{6} \varepsilon \tau^{1 / 2-1 / p}, & 2<p<\infty, \\
C_{7} \varepsilon \ln \left(\tau \varepsilon^{-2}\right)+C_{8} \varepsilon+C_{9} \varepsilon \tau^{1 / 2}, & p=\infty,\end{cases} \\
& \widetilde{\rho}_{p}(\varepsilon, \tau)= \begin{cases}C_{10} \varepsilon^{1-2 / p}, & 2<p<\infty, \\
C_{11} \varepsilon \ln \left(\tau \varepsilon^{-2}\right)+C_{12} \varepsilon, & p=\infty .\end{cases}
\end{aligned}
$$

Все постоянные здесь зависят лишь от $d, m, n, p,\|g\|_{L_{\infty}},\left\|g^{-1}\right\|_{L_{\infty}},\|Q\|_{L_{\infty}}$, $\left\|Q^{-1}\right\|_{L_{\infty}}, \alpha_{0}, \alpha_{1}$ и от параметров решетки $\Gamma$.

Отметим, что при фиксированном $\tau$ коэффициенты при $\|\mathbf{F}\|_{\mathscr{H}_{p}}$ в (12) и (13) суть $O\left(\varepsilon^{1-2 / p}\right)$ при $2<p<\infty$ и $O(\varepsilon|\ln \varepsilon|)$ при $p=\infty$. В аппроксимациях (12) и (13) содержится сглаживающий оператор (11) в отличие от случая однородной задачи Коши (ср. (6), (7)).

Выделим частные случаи, когда $g^{0}$ совпадает с $\bar{g}$ или с $\underline{g}$.

Замечание 5. 1. Равенство $g^{0}=\bar{g}$ равносильно соотношениям $b(\mathbf{D})^{*} \mathbf{g}_{k}(\mathbf{x})$ $=0, k=1, \ldots, m$, для столбцов $\mathbf{g}_{k}(\mathbf{x})$ матрицы $g(\mathbf{x})$. При его выполнении для периодического решения задачи (4) имеем $\Lambda(\mathbf{x})=0$ и корректоры в (6) и (12) обращаются в нуль.

2. Равенство $g^{0}=g$ равносильно следующим представлениям для столбцов $\mathbf{l}_{k}(\mathbf{x})$ матрицы $g(\mathbf{x})^{-1}: \mathbf{l}_{k}(\mathbf{x})=\mathbf{l}_{k}^{0}+b(\mathbf{D}) \mathbf{w}_{k}(\mathbf{x}), k=1, \ldots, m$, где $\mathbf{l}_{k}^{0} \in \mathbb{C}^{m}$, а $\mathbf{w}_{k}(\mathbf{x})$ суть Г-периодические функции из класса $H^{1}\left(\Omega ; \mathbb{C}^{n}\right)$. При его выполнении $\tilde{g}(\mathbf{x})=g^{0}=g$. Тогда $\tilde{g}^{\varepsilon} b(\mathbf{D}) \mathbf{u}_{0}=g^{0} b(\mathbf{D}) \mathbf{u}_{0}$ в $(7)$. Кроме того, в этом случае в (13) можно «устранить» $\Pi_{\varepsilon}$ и заменить $\tilde{g}^{\varepsilon} b(\mathbf{D}) \Pi_{\varepsilon} \mathbf{v}_{0}$ на $g^{0} b(\mathbf{D}) \mathbf{v}_{0}$. Эта замена отразится лишь на постоянных в оценке.

6. Метод исследования. Поясним метод исследования на примере более простой задачи (2), причем ограничимся обсуждением основной оценки (6). Представим матрицу $Q(\mathbf{x})^{-1}$ в виде $Q(\mathbf{x})^{-1}=f(\mathbf{x}) f(\mathbf{x})^{*}$, где $f(\mathbf{x})$ есть Г-периодическая $(n \times n)$-матрица-функция, такая, что $f, f^{-1} \in L_{\infty} . \mathrm{B} L_{2}\left(\mathbb{R}^{d} ; \mathbb{C}^{n}\right)$ рассмотрим дифференциальный оператор, формально заданный выражением $\mathscr{A}_{\varepsilon}=\left(f^{\varepsilon}\right)^{*} b(\mathbf{D})^{*} g^{\varepsilon} b(\mathbf{D}) f^{\varepsilon}$ (точное определение $\mathscr{A}_{\varepsilon}$ дается через соответствующую квадратичную форму).

Решение $\mathbf{u}_{\varepsilon}$ задачи (2) дается формулой

$$
\mathbf{u}_{\varepsilon}(\cdot, \tau)=f^{\varepsilon} e^{-\mathscr{A}_{\varepsilon} \tau}\left(f^{\varepsilon}\right)^{*} \phi .
$$

Поэтому дело сводится к изучению поведения окаймленной операторной экспоненты $f^{\varepsilon} e^{-\mathscr{A}_{\varepsilon} \tau}\left(f^{\varepsilon}\right)^{*}$ при малом $\varepsilon$. Решение $\mathbf{u}_{0}$ эффективной задачи (3) представляется в виде $\mathbf{u}_{0}=f_{0} e^{-\mathscr{A}^{0} \tau} f_{0} \phi$, где $f_{0}=(\bar{Q})^{-1 / 2}$ и $\mathscr{A}^{0}=f_{0} b(\mathbf{D})^{*} g^{0} b(\mathbf{D}) f_{0}$. В силу (14) оценка (6) непосредственно вытекает из следующего результата.

Теорема 6. При $\tau>0 u 0<\varepsilon \leqslant \tau^{1 / 2}$ справедлива оценка

$$
\left\|f^{\varepsilon} e^{-\mathscr{A}_{\varepsilon} \tau}\left(f^{\varepsilon}\right)^{*}-\left(I+\varepsilon \Lambda^{\varepsilon} b(\mathbf{D})\right) f_{0} e^{-\mathscr{A}^{0} \tau} f_{0}\right\|_{L_{2}\left(\mathbb{R}^{d}\right) \rightarrow H^{1}\left(\mathbb{R}^{d}\right)} \leqslant\left(C_{2} \tau^{-1}+C_{3} \tau^{-1 / 2}\right) \varepsilon .
$$


Положим $\widehat{\mathscr{A}_{\varepsilon}}=b(\mathbf{D})^{*} g^{\varepsilon} b(\mathbf{D})$. В существенном (15) сводится к неравенству

$$
\begin{gathered}
\left\|\widehat{\mathscr{A}}_{\varepsilon}^{1 / 2}\left(f^{\varepsilon} e^{-\mathscr{A}_{\varepsilon} \tau}\left(f^{\varepsilon}\right)^{*}-\left(I+\varepsilon \Lambda^{\varepsilon} b(\mathbf{D})\right) f_{0} e^{-\mathscr{A}^{0} \tau} f_{0}\right)\right\|_{L_{2}\left(\mathbb{R}^{d}\right) \rightarrow L_{2}\left(\mathbb{R}^{d}\right)} \leqslant \widehat{C} \tau^{-1} \varepsilon, \\
\tau>0,0<\varepsilon \leqslant \tau^{1 / 2} .
\end{gathered}
$$

С помощью масштабного преобразования оценка (16) непосредственно выводится из следующей теоремы.

Теорема 7. Пусть $\widehat{\mathscr{A}}=b(\mathbf{D})^{*} g b(\mathbf{D}), \mathscr{A}=f^{*} \widehat{\mathscr{A} f} . \Pi p u \tau \geqslant 1$ справедлива оиенка

$$
\left\|\widehat{\mathscr{A}}^{\mathrm{I} / 2}\left(f e^{-\mathscr{A} \tau} f^{*}-(I+\Lambda b(\mathbf{D})) f_{0} e^{-\mathscr{A}^{0} \tau} f_{0}\right)\right\|_{L_{2}\left(\mathbb{R}^{d}\right) \rightarrow L_{2}\left(\mathbb{R}^{d}\right)} \leqslant \widehat{C} \tau^{-1} .
$$

Приведенные редукции показывают, что гомогенизация для параболической задачи Коши тесно связана с вопросом о поведении окаймленной операторной экспоненты $f e^{-\mathscr{A} \tau} f^{*}$ при больших значениях $\tau$.

Далее, с помощью теории Флоке-Блоха оператор $\mathscr{A}$ разлагается в прямой интеграл по семейству операторов $\mathscr{A}(\mathbf{k})$, действующих в $L_{2}\left(\Omega ; \mathbb{C}^{n}\right)$ и зависящих от параметра $\mathbf{k} \in \widetilde{\Omega}($ квазиимпульса). Формально $\mathscr{A}(\mathbf{k})$ задается выражением $f^{*} b(\mathbf{D}+\mathbf{k})^{*} g b(\mathbf{D}+\mathbf{k}) f$ при периодических граничных условиях (точное определение дается через соответствующую квадратичную форму). Оценка (17) равносильна равномерному по параметру $\mathbf{k} \in \widetilde{\Omega}$ неравенству

$$
\left\|\widehat{\mathscr{A}}(\mathbf{k})^{1 / 2}\left(f e^{-\mathscr{A}(\mathbf{k}) \tau} f^{*}-(I+\Lambda b(\mathbf{D}+\mathbf{k})) f_{0} e^{-\mathscr{A}^{0}(\mathbf{k}) \tau} f_{0}\right)\right\|_{L_{2}(\Omega) \rightarrow L_{2}(\Omega)} \leqslant \widehat{C} \tau^{-1} .
$$

Здесь $\widehat{\mathscr{A}}(\mathbf{k})=b(\mathbf{D}+\mathbf{k})^{*} g b(\mathbf{D}+\mathbf{k}), \mathscr{A}^{0}(\mathbf{k})=f_{0} b(\mathbf{D}+\mathbf{k})^{*} g^{0} b(\mathbf{D}+\mathbf{k}) f_{0}$ (при периодических граничных условиях).

Оператор $\mathscr{A}(\mathbf{k})$ представляет собой эллиптический оператор в ограниченной области $\Omega$, аналитически зависящий от параметра k. Его спектр дискретен. Для анализа операторного семейства $\mathscr{A}(\mathbf{k})$ и доказательства оценки (18) мы применяем методы аналитической теории возмущений по одномерному параметру $t=|\mathbf{k}|$. Роль невозмущенного оператора играет $\mathscr{A}(0)$; точка $\lambda=0$ (край спектра) является изолированным собственным значением кратности $n$ для оператора $\mathscr{A}(0)$. Тогда при малом $|\mathbf{k}|$ вблизи нуля оператор $\mathscr{A}(\mathbf{k})$ имеет $n$ собственных значений, аналитически зависящих от $t=|\mathbf{k}|$. Асимптотические характеристики этих собственных значений и соответствующих собственных функций при малом $|\mathbf{k}|$ принято называть пороговыми характеристиками оператора $\mathscr{A}$ на краю спектра. Выясняется, что поведение окаймленной операторной экспоненты при больших значениях $\tau$ определяется пороговыми характеристиками оператора. В этом смысле можно говорить о том, что в параболических задачах (как и в эллиптических) гомогенизаиия является проявлением порогового эфбекта на краю спектра оператора $\mathscr{A}$.

Подробное изложение результатов, представленных в настоящей заметке, можно найти в [16].

\section{ЛиТЕРАТУРА}

[1] A. Bensoussan, J.-L. Lions, G. Papanicolaou, Asymptotic Analysis for Periodic Structures, Stud. Math. Appl., vol. 5, North-Holland Publishing Co., Amsterdam-New York, 1978. [2] Н. С. Бахвалов, Г. П. Панасенко, Осреднение прочессов в периодических средах, Наука, М., 1984. [3] В. В. Жиков, С. М. Козлов, О. А. Олейник, Усреднение дифференииальных операторов, Наука, М., 1993. [4] Е. В. Севостьянова, 
Матем. сб., 115:2 (1981), 204-222. [5] В. В. Жиков, Дифференциальные уравнения, 25:1 (1989), 44-50. [6] М. Ш. Бирман, Т. А. Суслина, Алгебра и анализ, 15:5 (2003), 1-108. [7] М. Ш. Бирман, Т. А. Суслина, Алгебра и анализ, 17:5 (2005), 69-90. [8] М. Ш. Бирман, Т. А. Суслина, Алгебра и анализ, 17:6 (2005), 1-104. [9] М. Ш. Бирман, Т. А. Суслина, Алгебра и анализ, 18:6 (2006), 1-130. [10] Т. А. Суслина, Функц. анализ и его прил., 38:4 (2004), 86-90. [11] Т. А. Suslina, in: Nonlinear Equations and Spectral Theory, Amer. Math. Soc. Transl. (2), vol. 220, Amer. Math. Soc., Providence, RI, 2007, 201-233. [12] Е. С. Василевская, Алгебра и анализ, 20:1 (2009), 3-60. [13] В. В. Жиков, Докл. РАН, 406:5 (2006), 597601. [14] V. V. Zhikov, S. E. Pastukhova, Russian J. Math. Phys., 12:4 (2005), 515524. [15] V. V. Zhikov, S. E. Pastukhova, Russian J. Math. Phys., 13:2 (2006), 224-237. [16] T. A. Suslina, Math. Model. Nat. Phenom., 5:4 (2010), 390-447.

Санкт-Петербургский государственный университет, Физический факультет

Поступило в редакцию e-mail: suslina@list.ru 26 апреля 2010 г.

Заведующая редакцией и научный редактор Г. М. Цукерман

Сдано в набор 24.09.2010. Подписано к печати 26.10.2010. Формат 70×100/16 Печать офсетная. Усл. печ. л. 7,8. Усл. кр.-отт. 1,9 тыс. Бум. л. 3,0 Уч.-изд. л. 8,0. Тираж 232 экз. Заказ 726.

Учредитель: Российская академия наук 